\title{
Two-dimensional Ultrasound Doppler Velocimeter for Investigations of Liquid Metal Flows
}

\author{
Franke, Sven; Lieske, Hendrik; Fischer, Andreas; Büttner, Lars; Czarske, Jürgen; Räbiger, Dirk; Eckert, \\ Sven*
}

Laboratory for Measurement and Testing Technologies, Faculty of Electrical Engineering and Information Technology, Technische Universität Dresden, Helmholtzstraße 18, 01069, Dresden, Germany

* Department Magnetohydrodynamics, Institute Safety Research, Forschungszentrum DresdenRossendorf, Bautzner Landstraße 128, 01328, Dresden, Germany

\begin{abstract}
A novel ultrasound Doppler array measurement system for flow investigations of electromagneticallydriven liquid metal flows is presented. The application of two linear ultrasound transducer arrays in an orthogonal arrangement facilitates the flow field measurement of the in-plane velocity components in a measuring plane of $67 \times 67 \mathrm{~mm}^{2}$ resolved into a grid of $24 \times 24$ vectors. Advanced processing techniques like the application of segmented arrays and the simultaneous operation of multiple elements of the transducer arrays allow high imaging frame rates and concurrently a high spatial resolution. Frame rates up to $30 \mathrm{frames} / \mathrm{s}$ according to a temporal resolution of $33 \mathrm{~ms}$ were achieved. Hence, the spatial structure of highly transient flow phenomena in non-transparent liquids can be investigated with high temporal resolution for the first time. The capabilities of the measurement system are demonstrated at a magnetic stirring model experiment at which liquid metal in a cubic vessel is driven by a rotating magnetic field.
\end{abstract}

\section{Introduction}

Metal and semiconductor industries strive permanently for higher productivities and better product qualities of processes involving metal and semiconductor melts. Innovative liquid metal technologies, in particular the optimized flow control by magnetic fields are developed continuously. A detailed knowledge of Lorentz-force driven multi-dimensional flow structures is required for understanding the interactions between liquid metal flows and different kinds of applied magnetic fields. Such investigations are often performed by means of numerical simulations. However, the underlying computer models of such simulations have to be validated by experimental investigations using efficient and reliable measurement techniques for liquid metal flows. Well-proven laser-based methods usually used for flow measurements in transparent fluids will obviously fail in opaque metal melts. However, the pulsed ultrasound Doppler velocimetry (UDV) [1] has proved as a reliable and attractive flow measurement technique for nontransparent fluids as liquid metals [2,3]. But it is hardly feasible to measure directly in real metal melts often with temperatures of over $1000^{\circ} \mathrm{C}$ since the ultrasound probe have to be in contact with the fluid flow to measure. Hence, flow investigations are performed at model experiments applying metals with melting points below room temperature such as mercury or the ternary liquid metal alloy GalnSn.

For extensive investigations of liquid metal flows occurring at temporal modulated AC magnetic fields instantaneous two-dimensional images of flow structure are exceedingly desirable. The current UDV technique provides instantaneous profiles of the velocity component aligned with the direction of ultrasound propagation. First attempts of a two-dimensional flow mapping by means of a consecutive electronic traversing with 20 single ultrasound transducers were realized [4]. However, the achieved measuring rates at most of 2 frames/s merely allowed to measure steady flows and the low number of ultrasound transducers considerably confined the spatial resolution of the flow structure.

We will present a novel ultrasound Doppler measurement system which overcomes these limitations providing a distinctly higher spatial and temporal resolution which makes it suitable for two-dimensional measurements of transient liquid metal flows.

\section{Measurement system}

The measurement system employs two identical linear ultrasound transducer arrays each comprising of 25 transducer elements spanning over a length of $67 \mathrm{~mm}$. The transducer elements measure the velocity component aligned with the direction of ultrasound beam. By the electronic traversing of the beam by means of the array elements the velocity component in a two-dimensional plane is gathered. The orthogonal arrangement of both arrays as shown in fig. 1a allows acquiring directly both in-plane flow velocity components in a measurement field of $67 \times 67 \mathrm{~mm}$.

The arrays are segmented into transducer elements of approx. $2.5 \times 5 \mathrm{~mm}^{2}$. During measurement two adjacent transducer elements are interconnected to operate as s single transducer with a size of 
$5 \times 5 \mathrm{~mm}^{2}$ effecting a low divergence of the ultrasound beam and with it a constant high lateral resolution over the entire measurement depth [5]. Consequently, the ultrasound beam can be shifted by one element size (by $2.5 \mathrm{~mm}$ ) in lateral direction at electronic traversing (fig. 1b) resulting in a measuring line pitch according to the lateral resolution in the focal point of the beam. The pairwise operation of the 25 transducer elements results in 24 measuring lines per array. The application of segmentation technique allows resolving smaller flow structures than achieved before with conventional UDV flow mapping of liquid metals.

The array transducers generate ultrasound waves at an emission frequency of $8 \mathrm{MHz}$ representing a compromise between a high penetration depth and a high spatial resolution. The ultrasound pulses typically consist of 8 harmonic wave cycles corresponding to an axial resolution of approx. $1.4 \mathrm{~mm}$ in the liquid metal GalnSn $\left(c_{\text {GalnSn }}=2740 \mathrm{~m} / \mathrm{s}\right)[6]$.

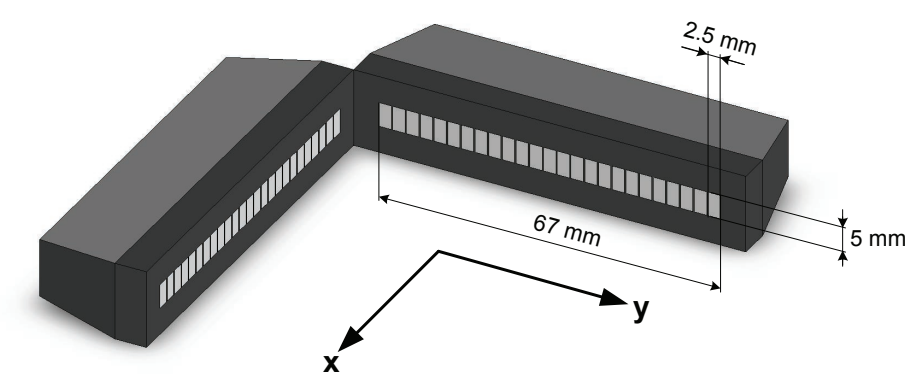

(a)

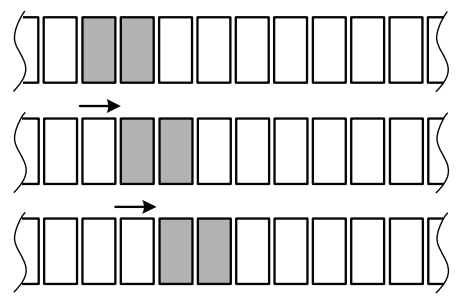

(b)

Fig. 1: Sensor design: (a) ultrasound array setup for vector field measurements and (b) pairwise operation and electronic traversing at segmental array technique

Previous approaches of multiline Doppler systems for the mapping of liquid metal flows applied plain sequential multiplexing to scan the measurement plane [4] inducing a low measurement rate of about 2 frames/s. However, for investigations of transient liquid metal flows driven by alternating magnetic fields two-dimensional flow mapping with high temporal resolution is required stringently. To overcome this problem several measuring lines are acquired simultaneously. In detail, instead of an exclusive sequential excitation of the array elements, as many transducer pairs as possible are driven in parallel. Thereby the problem of crosstalk has to be regarded which is caused by the overlap of the ultrasound beams due to their beam divergence. A sufficient distance between the transducer pairs being active at the same time minimizes possible crosstalk. For our current sensor array design previous experimental investigations revealed that a configuration with a spacing of 4 inactive transducer elements between the active transducer pairs induces a tolerable crosstalk of less than 1\% [6]. Based on the current array length of 25 transducer elements this spacing permits the parallel operation of four transducer pairs and accordingly the parallel acquisition of four measuring lines.

In the orthogonal configuration the transducer arrays have to be driven mutually since the tracer particles inside the fluid scatter the ultrasound beam in multiple directions causing crosstalk in the measuring lines perpendicular to the ultrasound beam.

The excitation pattern for the array control following the outlined processing principles above (as pairwise operation of transducer elements, parallel transducer operation and mutual array operation) is shown in fig. 2. The partly parallelized multiplex operation facilitates to scan all 48 measuring lines (24 measuring lines per array) in merely 12 time steps inducing to a considerable improved measurement rate. The excitation pattern is not sequenced linearly to additionally avoid the multiple excitation of the same transducer element in successive time steps which may induce temporal crosstalk by multiple pulse reflections between the vessel walls.

The block diagram of the measurement system is depicted in fig. 3 . The main component is the multiplex circuit realizing the excitation pattern. The trigger control actuates the burst generators (an arbitrary function generator (AFG) with a RF amplifier) to generate a high-voltage sinusoidal burst signal. This burst signal is directed to the active transducers of the respective time step (cp. Fig. 2) by fast electronic switching matrixes [7]. Their switch configurations are obtained from the multiplex control according to the excitation pattern. The active transducers excite the ultrasound pulses and receive the particular echo signals. Afterwards the signals are amplified and directed to the output channels of the switching matrix. For processing these signals are recorded by data acquisition cards in a computer. The software-based offline signal processing in the computer is performed with MATLAB. The signal processing for determining the Doppler frequency is based on the estimation of phase shift between two pulse echoes. To obtain suitable images of flow fields and accordingly low measurement uncertainties the mean 
Doppler frequency is estimated from a set of consecutive pulse echoes, typically 25 to 100 . A phase shift estimator according to the autocorrelation technique [8] was applied.

The maximum repetition frequency of the excitation pattern amounts to $1.5 \mathrm{kHz}$ in GalnSn [6]. Assuming a typical number of 50 pulse echoes per flow map measurement rates up to 30 frames/s can be achieved representing a distinct improvement in temporal resolution.

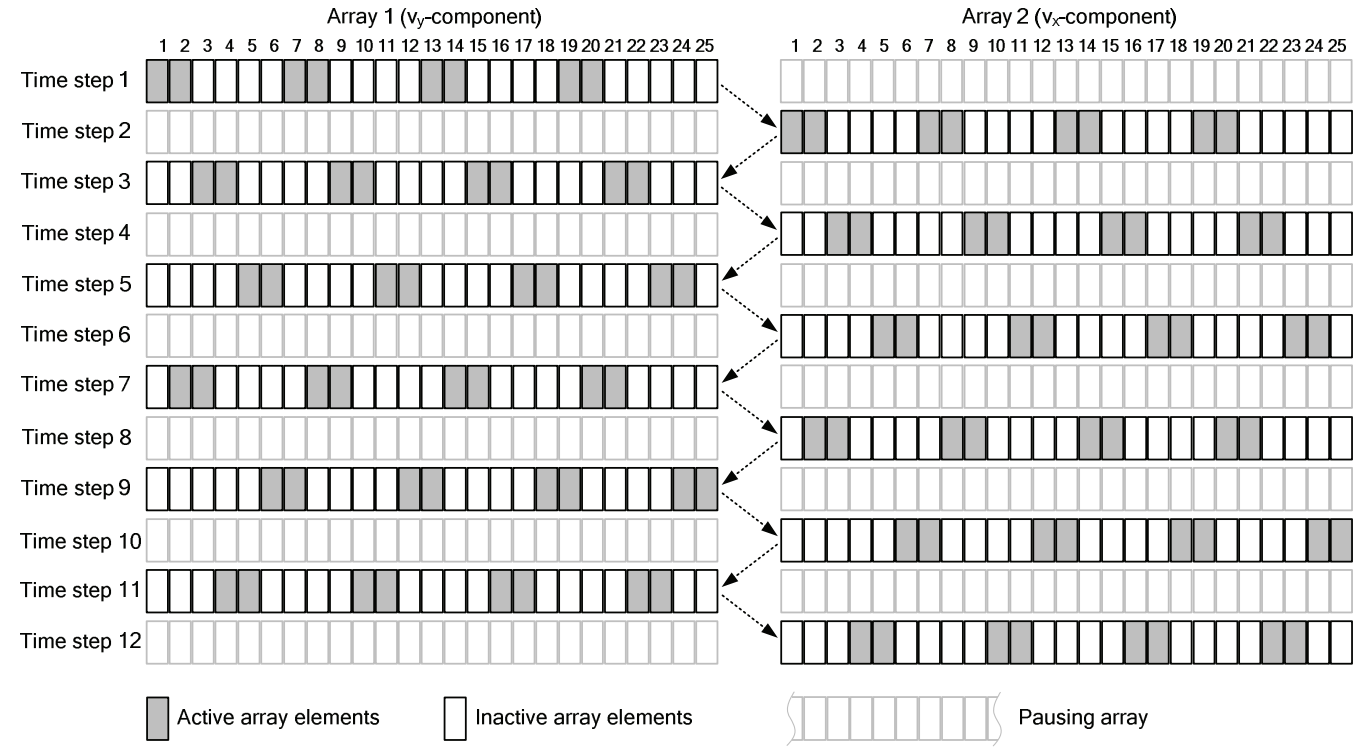

Fig. 2: Excitation pattern of mutual array operation at orthogonal array configuration

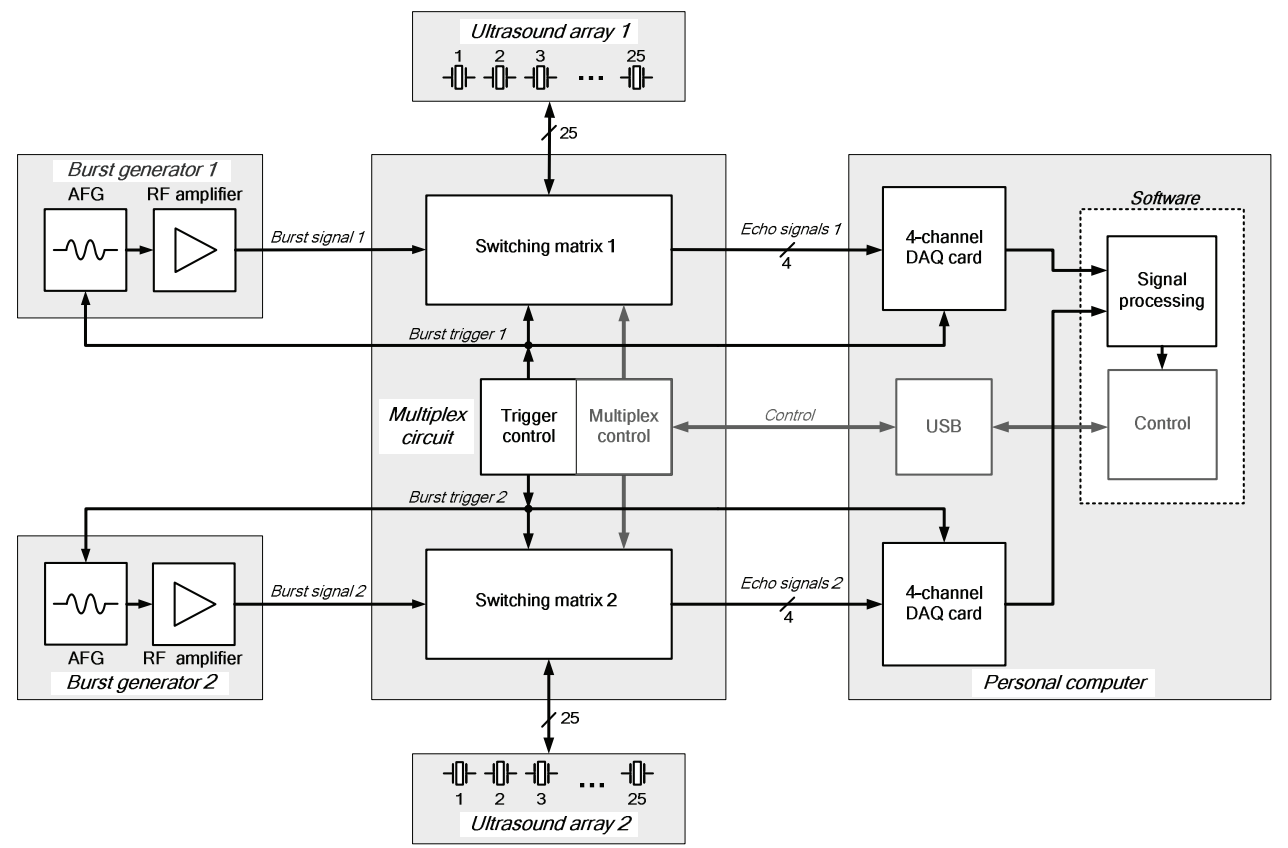

Fig. 3: Block diagram of ultrasound Doppler velocimeter for two-dimensional flow mapping

\section{Experimental setup}

A basic issue at investigations of magnetic flow control involves the mixing of metal melts by means of magnetic stirrer systems. In our experiments a rotating magnetic field stirrer was applied to demonstrate the capabilities of the measuring system at a real liquid metal flow. A schematic view of the experimental setup is depicted in fig. 4. Six coils are arranged in a pole-pair connection to create the rotating magnetic field (RMF). The bore diameter of the magnetic system is $200 \mathrm{~mm}$, wherein a cubic fluid vessel with an interior edge length of $75 \mathrm{~mm}$ was placed concentrically. The RMF drives the liquid metal (GalnSn) inside the fluid vessel in a swirling flow with a rotation direction equal to the inducing magnetic field. The physical principle driving the rotating flow is similar to the operation principle of an asynchronous motor. Similar to the asynchronous motor a slip between inducing field and generated flow occurs. 
The ultrasound arrays are mounted horizontally at half the height of the vessel walls to measure the swirling flow in the horizontal mid-plane of the vessel (fig. 5a). However, the mid-plane $\left(75 \times 75 \mathrm{~mm}^{2}\right)$ is not covered completely by the measurement field $\left(67 \times 67 \mathrm{~mm}^{2}\right)$ of the arrays due to the structural limitations of the sensor arrays.

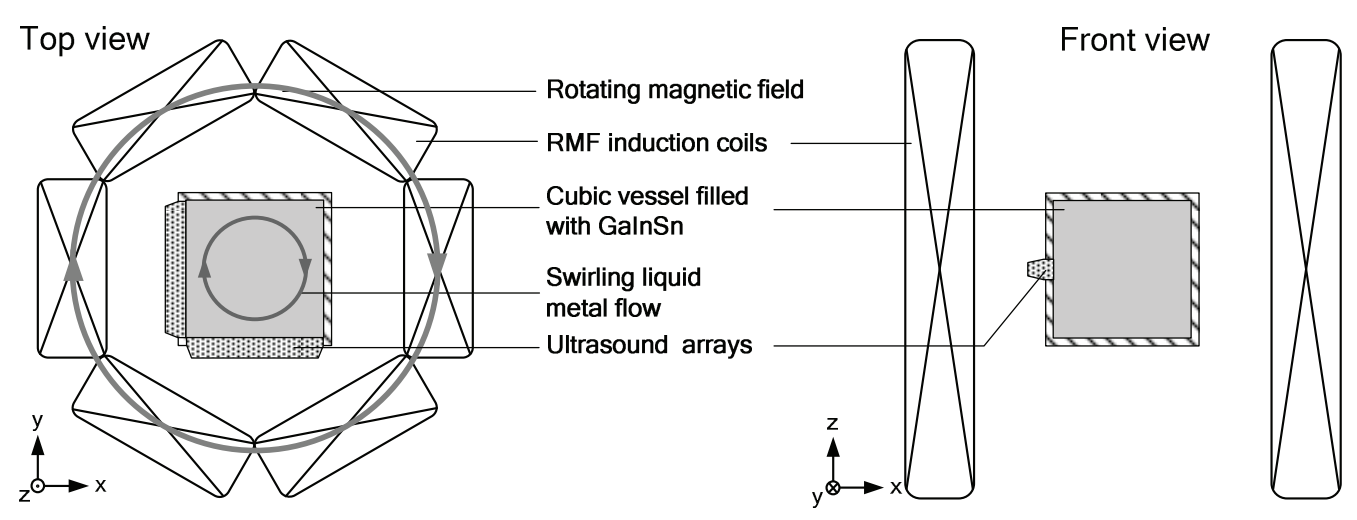

Fig. 4: Experimental setup: GalnSn in a plexiglas cube driven by a rotating magnetic field stirrer

\section{Measurement results}

In a first experiment a stationary rotating magnetic field with a field strength of $B_{0}=4.8 \mathrm{mT}$ at an angular frequency $\omega_{\text {RMF }}=2 \pi \cdot 50 \mathrm{~Hz}$ was applied indicating a fully developed turbulent flow in the experiment [9]. The measurements were performed using a pattern repetition frequency of $1.5 \mathrm{kHz}$ with an averaging about 50 echo signals leading to a temporal resolution of $\Delta \mathrm{T}=33 \mathrm{~ms}$ according to a frame rate of 30 frames/s. Figure $5 \mathrm{~b}$ depicts a snapshot of the flow showing a clock wise rotating vortex according to the rotation direction of the magnetic field (cp. Fig. 5a). The slip amounts to about $99 \%$. In the figure the center of the vortex does not coincide exactly with the cube center. Our observations revealed that the center of the main vortex fluctuates about $5 \mathrm{~mm}$ around the cube axis due to the turbulent characteristic of the flow.

Figure $5 \mathrm{~b}$ shows some artifacts (as misorientation of several vector arrows) occurring at various positions within the flow field. These artifacts may arise from a temporary and local deficit of scattering particles or a low signal-to-noise-ratio leading to high measurement uncertainties.

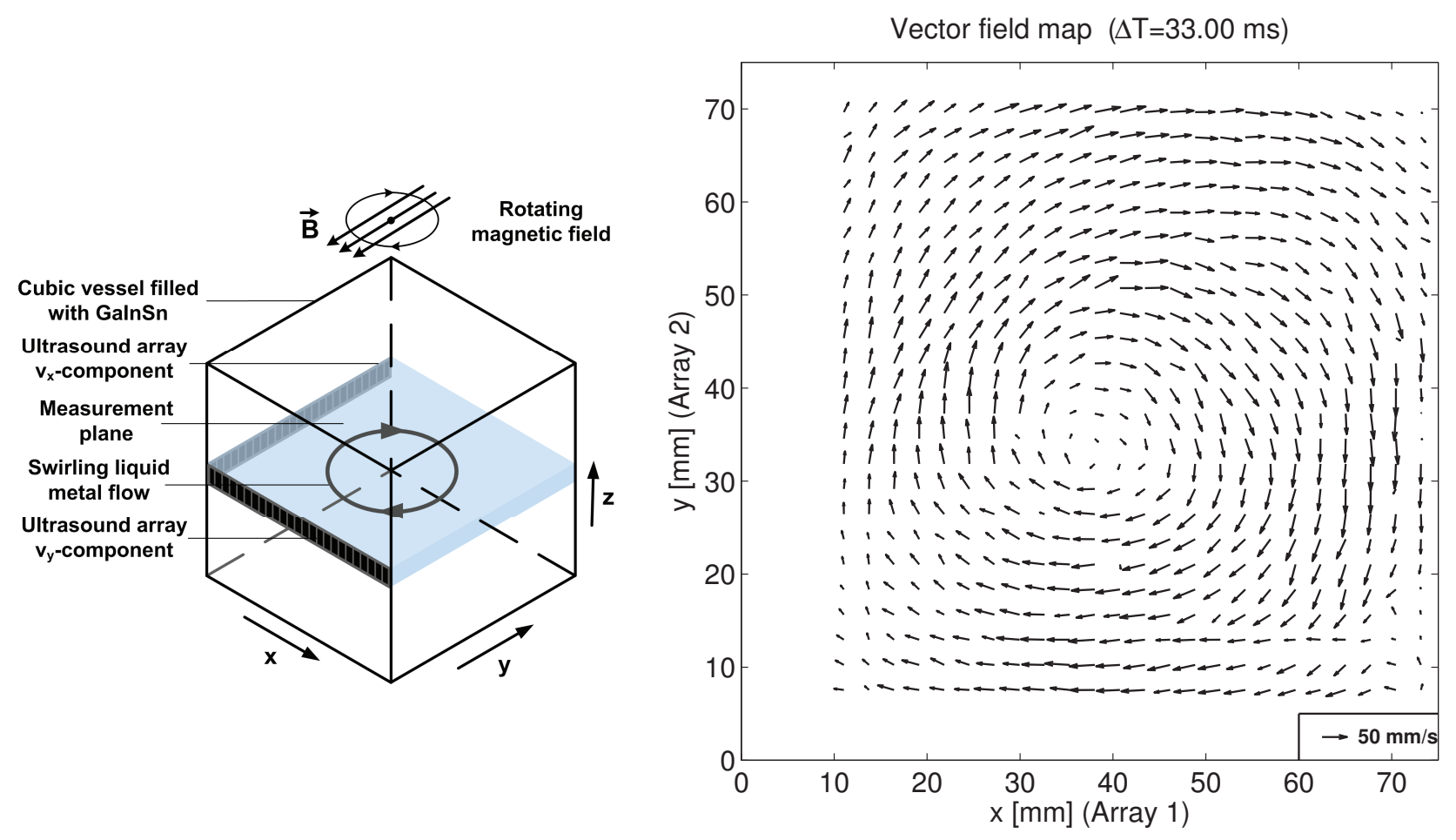

(a)

(b)

Fig. 5: Magnetic stirring: (a) Sensor setup and (b) respective measurement result with $\Delta T=33 \mathrm{~ms}$ 
(a)

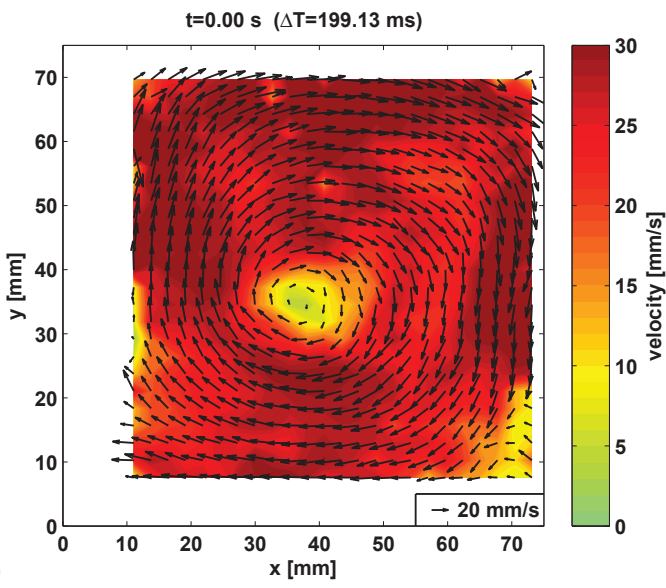

(c)

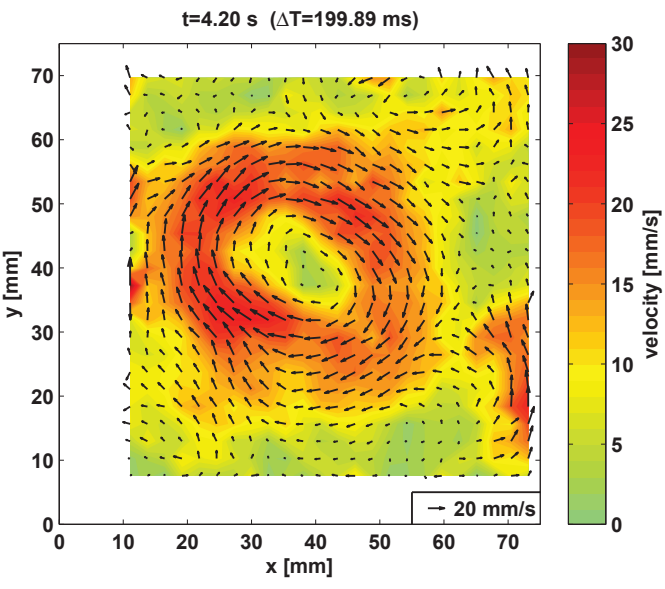

$\mathrm{t}=9.01 \mathrm{~s} \quad(\Delta \mathrm{T}=199.89 \mathrm{~ms})$

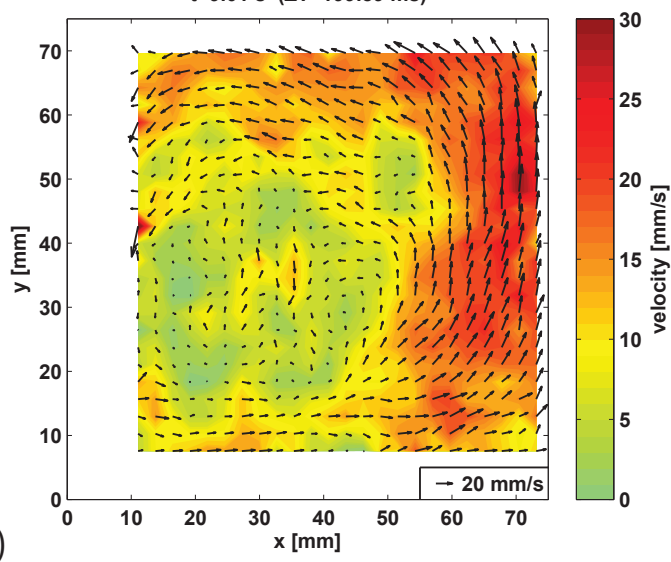

$\mathrm{t}=19.21 \mathrm{~s}(\Delta \mathrm{T}=199.89 \mathrm{~ms})$

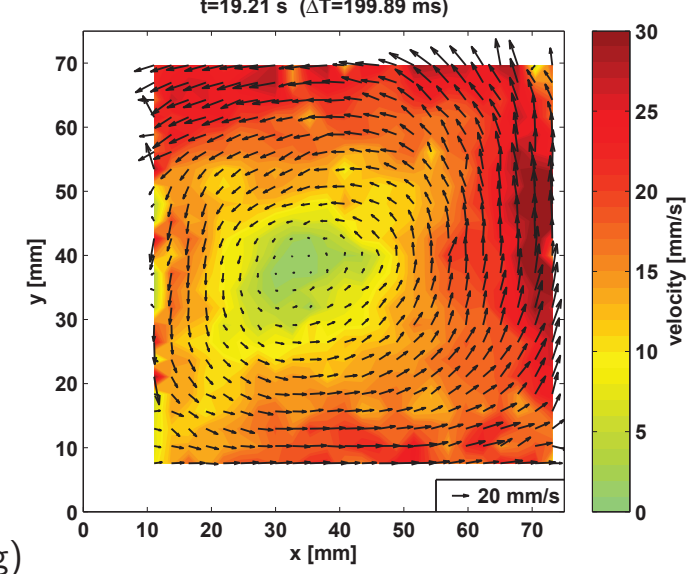

(b)
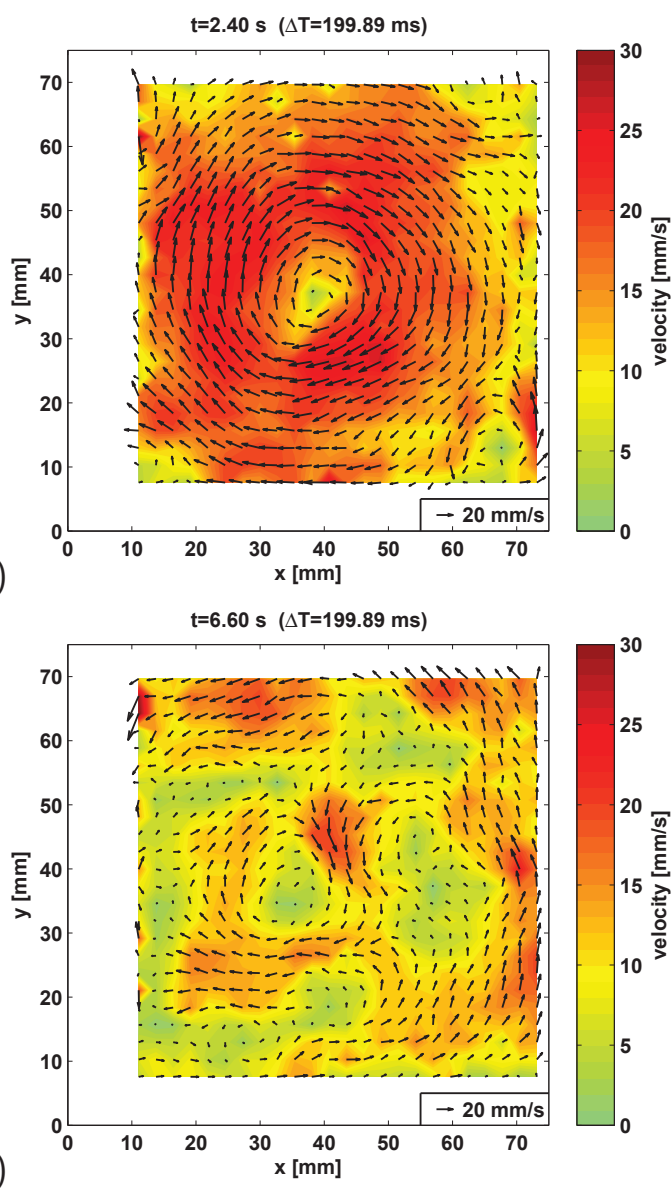

(d)

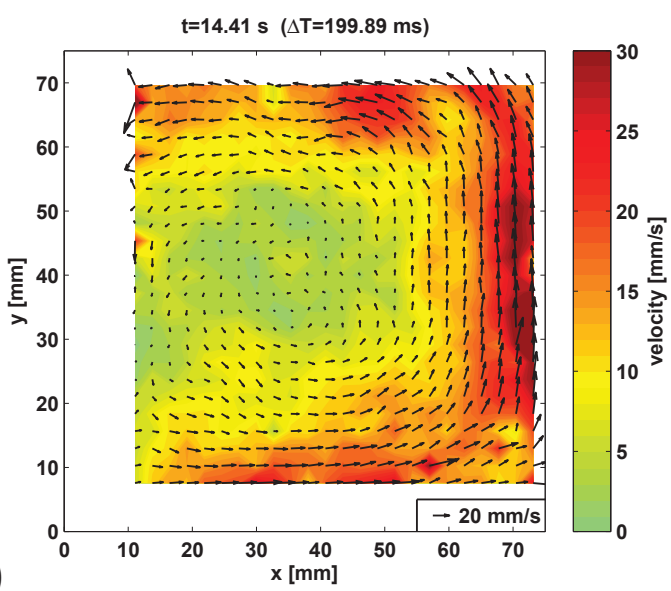

(f)

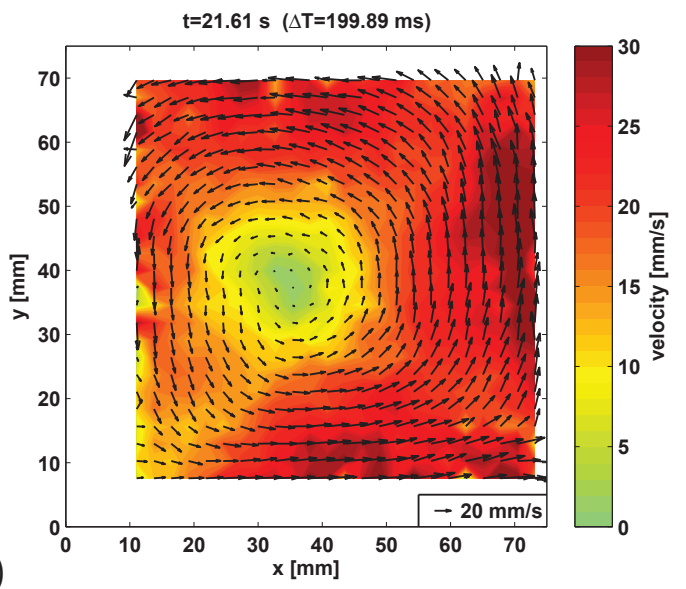

Fig. 6: Measured sequence of flow fields after reversal of rotating direction of magnetic field at time $t=200 \mathrm{~ms}$ 
A continuous stirring of metal melts by constantly rotating magnetic fields often provides only an insufficient and ineffective mixing. The use of RMF pulse sequences of alternating direction proved to be an efficient technique to overcome this problem [10]. The capabilities of our measuring system to reveal transient flow structures occurring at alternating rotating magnetic fields predestines it for investigating such processes.

A selected sequence of such a transition procedure of the RMF is shown in fig. 6 . A magnetic field strength of $B_{0}=3.1 \mathrm{mT}$ was selected in this experiment. The measurement was started just $200 \mathrm{~ms}$ before the direction of the RMF was reversed. The measurements were performed at a pattern repetition frequency of $500 \mathrm{~Hz}$ using an averaging about 100 pulse echoes for low measurement uncertainty. This induces a temporal resolution of $200 \mathrm{~ms}$.

Figure 6a shows the initial state of the steady flow before the reversal of rotation direction. The first significant changes in the flow structure (fig. 6b) arise in the exterior regions of the cross section where the magnetic force achieves its maximum. Until about $4 \mathrm{~s}$ after reversal (fig. $6 \mathrm{c}$ ) a residual core rotating in the primary direction is observed whereas near the walls no explicit flow structure exists. Figure $6 \mathrm{~d}$ presents a time period without dominating vortex but with a transient ensemble of several vortices. Beginning with fig. $6 e$ the original vortex structure is collapsed completely and a vortex flow in the opposite rotation direction appears in the regions nearby the walls. The figures $6 f-6 \mathrm{~h}$ document the ongoing strengthening of the central vortex.

\section{Summary}

We presented an imaging flow measurement technique based on the ultrasound Doppler velocimetry for the investigation of transient liquid metal flows. The measuring system employs two linear ultrasound arrays in an orthogonal sensor configuration to capture both in-plane velocity components of flow in the field spanned by the two arrays. The field covers a plane of $67 \times 67 \mathrm{~mm}^{2}$ resolved into a grid of $24 \times 24$ vectors. Advanced processing techniques like the parallel operation of multiple transducers and the segmentation principle of the ultrasound arrays facilitate a high spatial as well as a high temporal resolution of the measurements overcoming the present UDV techniques for a two-dimensional flow mapping by far. Measurements were performed in order to verify the capabilities of the system at a respective model experiment applying the liquid metal GalnSn driven by a rotating magnetic field. The measurements demonstrated measuring rates up to 30 frames/s. The system allowed performing temporally and spatially highly resolved measurements of transient flows for the first time compared to previous multiline ultrasound Doppler systems proven at a RMF-driven flow with reversal of rotation direction.

A future aspect will be the extension of the UDV system to provide three-dimensional visualizations of turbulent liquid metal flows. The measurement system will be applied at various experiments with liquid metals or other non-transparent flows.

\section{References:}

[1] Takeda Y: Development of an ultrasound velocity Profile monitor, Nucl. Eng. Design 126 (1991), 277-284.

[2] Eckert S, Cramer A, Gerbeth G: Velocity measurement techniques for liquid metal flows, in "Magnetohydrodynamics - Historical Evolution and Trends", Molokov S, Moreau R, Moffatt HK (Eds.), Springer-Verlag, Dordrecht (2007), 275-294.

[3] Cramer A, Zhang C, Eckert S: Local flow structures in liquid metals measured by ultrasonic Doppler velocimetry, Flow Measurement and Instrumentation, Volume 15, 2004, p. 145-153

[4] Takeda Y, Kikura H: Flow mapping of mercury flow, Exp. Fluids 32 (2002), 161-169.

[5] Hedrick WR, Hykes DL, Starchman DE: Ultrasound Physics and Instrumentation, Elsevier Mosby, St. Louis (2005), 4 ed.

[6] S. Franke, L. Büttner, J. Czarske, D. Räbiger, S. Eckert: Ultrasound Doppler system for two-dimensional flow mapping in liquid metals, Flow Measurement and Instrumentation, Volume 21, 2010, p. 402-409.

[7] S. Franke, H. Lieske, A. Fischer, L. Büttner, J. Czarske, D. Räbiger, S. Eckert: 2d-2c Ultrasound Doppler Array Velocimeter for Flow Investigations in Liquid Metals, 7th International Symposium on Ultrasonic Doppler Methods for Fluid Mechanics and Fluid Engineering, April 07 - 09, 2010, Göteborg, Sweden, p. 89 - 92.

[8] Kasai C, Namekawa K, Koyano A, Omoto R: Real-time two-dimensional blood flow imaging using an autocorrelation technique, IEEE Trans. Sonics. Ultrason. 32 (1985), 458-464.

[9] Frana K, Stiller J: A numerical study of flows driven by a rotating magnetic field in a square container, Eur $\mathrm{J}$ Mech B Fluid 27 (2008), 491-500.

[10] Eckert S, Nikrityuk PA, Räbiger D, Eckert K, Gerbeth G: Efficient melt stirring using pulse sequences of a rotating magnetic field: Part I. Flow field in a liquid metal column, Metall. Mater. Trans. 38B (2007), 977-988. 